\title{
APPLING REMOTE SENSING TECHNIQUE TO MONITORING SPATIAL EXPANSION OF IMPORTANT CITIES IN CHINA-INDOCHINA PENINSULA ECONOMIC CORRIDOR
}

\author{
Li Jiayu ${ }^{1,2}$,Wang Huabin ${ }^{1,2}$,Wang Guanghui ${ }^{2}$,Zhai Haoran ${ }^{2}$, Han Min $^{1,2}$, Liu Xiao $^{2}$ \\ 1. Liaoning Technical University, Fuxin 123000, China (sas_ljy@163.com) \\ 2. Satellite Surveying and Mapping Application Center, Beijing 100048, China
}

\begin{abstract}
KEY WORDS: China-Indochina Peninsula Economic Corridor, Random forest, Impervious surface, Urban expansion, Landscape pattern metrics
\end{abstract}

\begin{abstract}
:
Since twentieth Century, the process of economic globalization has made great progress, and Southeast Asia has developed rapidly under the background of international industrial transferring. In this paper, the 6 important nodes cities in China - Indochina Peninsula along the economic corridor are took as study area. The main data is time series Landsat data. The method of object-oriented random forest classification was used to extract the classification results of study area from 2000 to 2015 . The urban expansion of the node cities was evaluated by calculating the expansion speed of the impervious surface and the landscape pattern metrics. The results indicated that the method of object oriented random forest classification can effectively extract the urban impervious surface. the overall accuracy is over $81 \%$, and the Kappa coefficient is over 0.82 . In the past 15 years, the expansion speed of Vientiane city was fastest in 6 countries. The area of urban impervious surface expanded 8 times than that of 2000.The pattern of expansion is summarized as "gather first-diffuse then", "diffuse first-gather then" and "gather". Overall, the process of urbanization of these cities are still in the rising period.
\end{abstract}

\section{INTRODUCTION}

In 2013, the Chinese government has proposed to build The Silk Road Economic Belt and the 21st-century Maritime Silk Road (The Belt and Road Initiative, B\&R). The Belt and Road throughout Africa and Europe, East, holding the vigorous development of the Asia Pacific Economic Circle, the European economic circle of the west developed countries, the middle of the hinterland economic development potential. It takes the cities along the belt as basis. It aims to build several economic corridors such as Mongolia and Russia, the new Eurasian Continental Bridge, Chinese - Asia - West, China - Indochina, Pakistan and BCIM economic corridor by establishing cooperation with the key economic and trade industrial areas along the B\&R. While building the framework, infrastructure construction is one of the most important part. The spatial spill over effect produced by the interaction between local infrastructures and frame infrastructures, which reduces the inter city traffic geographic economic gap formation, and promotes the convergence of different economic conditions, has significant boost the regional economic development. The industrials along the B\&R cluster into many groups, Which rise the employment rate of the vicinities (Xueping Liu,2015). With rapidly developing of urban economics along the $B \& R$, the extensive industry often produces serious environmental problems such as air pollution, water pollution and so on. Therefore, it is necessary to study the urbanization mode along the economic corridor in order to Direct the developing of urban economy in an environmental-friendly way.

Most of the theories of the evolution of urban development is based on the internal logic of the theory of large cities leading, which is based on economies of scale and economies of scope (Ingram G K, 1997). The area of impervious surface is considered to be an important indicator of the city expansion, environmental change and economic development. Deng $\mathrm{Y}$ analysed the changes of impervious layer area in Pearl River Delta, which is calculated by the images of impervious layer. The image is generated by a linear spectral unmixed method using the Landsat images of this area (Deng Y,2012). Taubenböck analysed the footprint of 12 mega cities' expansion during $1975-2010$ by object-oriented decision tree using the Landsat imagery and Terra SAR data (H. Taubenböck, 2012). Zang $S$ et al analysed three cities of Harbin, Daqing, and Qiqihar during 1990-2005 the decline of ecosystem service value caused by land use change by using mixed supervision and unsupervised classification (Zang S, 2010). Shi L used the multi-source remote sensing image in Shenzhen, Xiamen, 
Haikou from 1973 to 2013 as an example, selected the expansion rate and expansion of the city, influence on land use form of city compactness and city centre migration as index, combined with the natural elements and human factors on the characteristics of space economic zone city expands when analysing (Shi L, 2015). Che Q analysed the urban spatial expansion and evolution of diversity, heterogeneity and spatial organization of "hot spots" pattern in the Yangtze River Delta during 1985-2007. At the same time, he analysed the driving force to the development of the city (Che Q, 2011). By comparing results of domestic researches and foreign researches, we found that most eastern countries and Western countries are at the different development stage of urbanization, which because of the economic, cultural and political factors. Therefore, an empirical study which is based on the case of long time series of urban space and environment evolution can enhanced our understanding of urban evolution characteristics and development law, which has important significance for maintaining regional ecological security and development of the cities (Wang L, 2016).

In this paper, we use the time series of Landsat data to extract urban land use results of the 6 important node cities along the economic corridor between China and Indochina Peninsula from 2000 to 2015 by an object-oriented Stochastic Forest Classification method. On the basis of the calculation of the spatial expansion rate and landscape pattern metrics of urban impervious surface, this paper makes a comprehensive analysis of the spatial evolution patterns of each node and give a reasonable evaluation of its development.

\section{RESEARCH AREA AND DATA SOURCES}

\subsection{Overview of the study area}

China-Indochina Peninsula economic corridor is one of the six major economic zones and is also the China and the B\&R neighbouring countries jointly planned construction. The corridor on the trans Asian Railway, starting in Yunnan Kunming and Guangxi Nanning, across Vietnam, Hanoi, Phnom Penh, Bangkok, Kuala Lumpur to Singapore, is connected China Indochina continental bridge, but also Chinese cooperation with ASEAN transnational economic corridor. The 6 important node cities along the economic corridor of the China-Indochina Peninsula were selected as the research objects, including Vientiane, Hanoi, Phnom Penh, Bangkok, Kuala Lumpur and Singapore. Singapore is a developed city. Other cities are developing countries. The above cities are their country's political, economic and cultural centre. These cities have the excellent geographical location, abundant natural resources and huge potential for economic development. These are the key hub of China trade cooperation in Southeast Asia.

\subsection{Data and pre-processing}

Land classification data we used six cities in 2000, 2005, 2010 and 2015 Landsat MSS/TM/ETM+/OLI data. The accuracy verification data we used Google Earth high resolution data during 2000-2015. The specific use of remote sensing image is shown in Table 1. Image pre-processing includes image registration, radiometric calibration, atmospheric correction and clipping.

\begin{tabular}{|c|cccc|}
\hline City & 2000 & 2005 & 2010 & 2015 \\
\hline Vietnam & TM 2000 & TM 2005 & TM 2011 & OLI 2015 \\
Hanoi & TM 2000 & TM 2005 & TM 2010 & OLI 2016 \\
Phnom Penh & TM 2000 & TM 2005 & TM 2009 & OLI 2015 \\
Bangkok & TM 2000 & TM 2005 & TM 2009 & OLI 2015 \\
Kuala Lumpur & TM 2000 & TM 2006 & TM 2010 & OLI 2015 \\
Singapore & TM 2000 & TM 2005 & TM 2009 & OLI 2015 \\
\hline
\end{tabular}

Table 1. Data used in experimentation area

\section{RESEARCH METHODOLOGY}

Based on the data of Landsat, we analysed the situation of urban development of the 6 node cities in China - Indochina Peninsula economic corridor after 2000, and the specific process is shown in figure 2. The Landsat satellite data pre-processing be taken first. Land classification results in the study area were obtained by multiscale segmentation and random forest classification, then the classification results are evaluated. if the accuracy is too low, then Selecting samples for classification again. Finally, through the calculation of landscape pattern metrics and the rate of urban expansion, we analysed the expansion of urban impervious surface. 


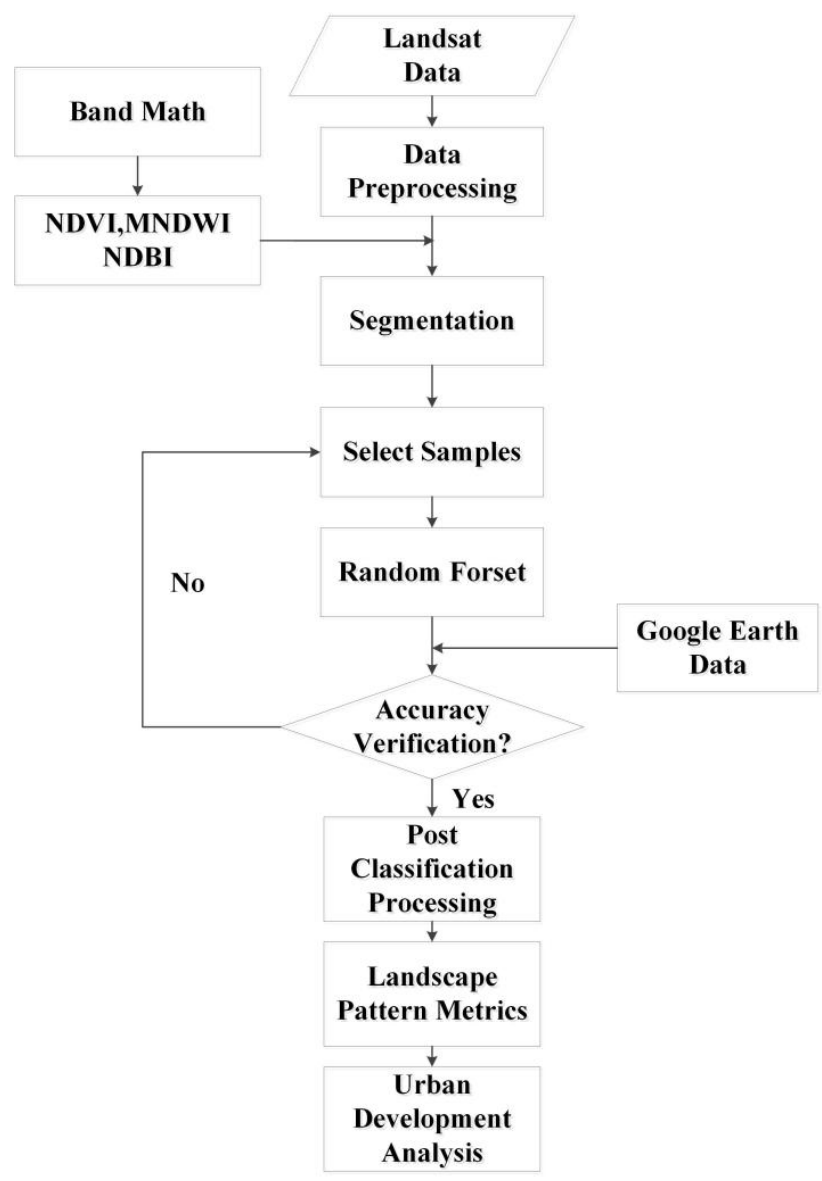

Figure 2. Technical flow chart

\subsection{Multiscale segmentation}

Segmentation is the merging of adjacent pixels of the same or similar features into an object. Segmentation scale determines the maximum heterogeneity allowed by the generated image object. In the scale parameter selection, we should try to ensure that each image object contains only a class of objects, and strive to segment the image object size is appropriate, not broken (Liu D, 2010).In this paper, we tested in the scale range of 30-100 in the study area, the optimal segmentation scale selection of the study area through visual interpretation of the way, the final measured Landsat TM data scale is in the vicinity of 40 , the OLI data standard is in the vicinity of 60.The layer weight represents the proportion of each band in the segmentation. In order to make full use of the information of each band and balance the effect of each band in the segmentation process, the weight of each band is set to 1 .

The homogeneity criteria include shape factor and compactness factor. The shape factor represents the weight of the shape standard in segmentation. The sum of the shape factor and the spectral feature is 1 . The greater the value of the shape factor, the smaller the influence of the spectral characteristics of the image in the segmentation process. In the process of segmentation, spectral features often play a greater role. As a result, the spectral features are usually given greater weight to better separate the different objects. Smoothness determines the smoothness of the edge of the image sample. The sum of the smoothing factor and the compactness factor is 1 . The smoother the image samples are, the lower compactness is. Through many experiments, the range of shape factor and compactness factor are $0.1-0.3$ and $0.5-0.7$, respectively.

\subsection{Random forest classification}

Random forest is a kind of machine learning. It is a kind of supervised classification method which is widely used in remote sensing. The concept of random forest was first proposed by Breiman Leo in 2001 (Breiman, 2001).The decision tree classification method based on repeated two point operation is combined to form random forest. The decision tree is grown by the random selection vector, and each tree will grow completely without pruning. And when the decision tree is generated, each node is divided into several variables randomly selected. After all the decision trees are generated, we use the voting method to classify the results of all decision trees (Guo Y, 2016).

Due to the randomness of the data set and the characteristic variable and the independence of the decision tree, the random forest classification method has the characteristics of anti-noise, not easy to fit, fast training speed, no feature selection and so on. The prediction accuracy of random forest is improved under the condition of no significant increase in computation. Random forests are not sensitive to multicollinearity, the results are robust to missing data and imbalanced data, which is regarded as one of the best algorithms.

In the application of random forest classification process, in the case of a certain number of training samples, the impact of the classification accuracy of the two main parameters: the maximum number of features (max_features) and the maximum number of random trees. The size of max_features is related to the ability of decision tree and the correlation between decision trees. The larger the max_features is, the better the performance of the model can be. At each node, we will have more choices to consider, and the classification ability of a single decision tree is larger. At the same time, it also results in the increase of the correlation among decision trees, which reduces the classifier ability. Therefore, the max_features optimal parameters can be adjusted according to the out of bag(OOB) error rate to achieve higher accuracy (Wolpert,1999). The maximum number of random trees is the number of decision trees when the classification is completed. More subtrees can make the model have better performance. Breiman (1996) proved that based on the large number theorem, when the maximum number of random trees increases, the model generalization error converges (Breiman,1999). So we do not need to worry about overtraining. Therefore, under the premise of memory, select the maximum number of random trees as many as possible. The classification of the maximum number of tree classification is set to 200. Other parameters are set by default. 
According to the V-I-S model (RIDD, 1995), the land cover types were divided into impervious surface, vegetation, bare land and water. According to the principle of random and uniform, 200 objects were selected as training samples. According to these finite samples, we can find the best compromise between model complexity and learning ability. In this study, NDVI, MNDWI, NDBI and other spectral bands, spectral features, spatial features and texture features are used to participate in random forest classification.

\subsection{Post classification processing}

Although supervised classification can quickly carry out land classification, but due to the difference in spectral features of different images and image area samples in the classification process inevitably caused some wrong leakage points. In order to analyse the land use change quantitatively, it is necessary to modify the classification results. Classification postprocessing refers to sequentially modify manually and logical modification of random forest classification results.

Urban impervious surface is an important indicator of urban expansion. In the expansion of the development process, the trend generally meet the increased year by year, from the law of development. Therefore, according to the logic, we can infer that the location of a certain position in the current year is impermeable, and the same position in the following year is also an impermeable surface. Manual correction is a visual correction of the wrong points, according to the Google Earth on the same period high-resolution image interpretation.

\subsection{Spatial expansion intensity index}

The spatial expansion velocity (V) is used to describe and analyse the expansion speed of urban agglomeration space in different periods, which is mainly defined by the expansion area of urban space, the research period and the total area of the research unit (Wang L, 2010).

$$
V=\frac{-\Delta U_{i} \times 100 \%}{U_{L} \times \Delta t}(1)
$$

Where $\Delta \mathrm{U}_{\mathrm{i}}$ represents the study period of unit area of urban space expansion; $\Delta t$ is the study period of time; $U_{L}$ is the initial stage of a period of impervious surface area.

\subsection{Urban landscape pattern metrics}

Landscape spatial pattern mainly refers to the spatial arrangement of landscape patches with different sizes and shapes. It is an important manifestation of landscape heterogeneity, reflecting the effect of various ecological processes on different scale (Pan D, 1999). the landscape pattern is the formation of natural and artificial factors in the region. Landscape pattern is formed by natural factors and human factors in a certain area. From seemingly out of order of land cover patches, found that the distribution of inner space is the significance of landscape pattern analysis. We use fragstats4.2 software to calculate the landscape pattern metrics, and we select the largest patch index (LPI), Euclidean nearest neighbour distance Index (ENN), Landscape Division Index (DIVISION) and Splitting Index (SPLIT) to analyse the dynamic change of landscape pattern along the city China Indochina Peninsula Economic zone. LPI is equal to the proportion of the largest block in a block type to occupy the whole landscape area. In this study, the bigger the LPI, the more obvious the landscape dominance of impervious surface is. The ENN is the distance from the nearest neighbour patch, which reflects the spatial distribution of land use landscape patches. The expansion of the patch area often leads to the decrease of ENN, but the increase of ENN is caused by the aggregation of the patches with a relatively short distance. The larger the ENN, the more shrinkage of the spatial distribution. The degree of separation refers to the spatial distribution of individual patches in different landscape type (Chen L, 2001). DIVISION is equal to the square and the area divided by 1 minus all the plaques of the entire landscape area. $0<$ DIVISION $<1$. DIVISION is used to analyse the spatial distribution characteristics of impervious surface. the greater the DIVISION, the more discrete the impermeable surface. SPLIT is the sum of the square of the landscape area divided by the square of all types of patches. When the landscape consists of a patch, SPLIT=1.Its value increases with the further subdivision of the landscape, and the maximum value of SPLIT is reached when the landscape is maximized. SPLIT is used to analyse the degree of fragmentation of impervious landscape in the study area. The larger the SPLIT value, the more broken the landscape.

\section{RESULTS AND ANALYSIS}

\section{1 precision evaluation}

We adopt the method of randomly selecting the sample points to verify the accuracy of the corresponding image in Google Earth. The number of selected points for each landscape is 400 , of which the impervious surface, bare land, vegetation, water samples of the number of 100 . The confusion matrix of the classification results of six cities in the past 4 years is obtained by the method of point by point visual interpretation, and the overall accuracy (OA) and Kappa coefficient are calculated. Accuracy evaluation results are shown in table 3.

\begin{tabular}{|cc|cccccc|}
\hline \multicolumn{2}{|c|}{ CITY } & Vietnam & Hanoi & Phnom Penh & Bangkok & Kuala Lumpur & Singapore \\
\hline 2000 & OA & $83.40 \%$ & $82.50 \%$ & $85.40 \%$ & $89.60 \%$ & $86.20 \%$ & $91.20 \%$ \\
\hline
\end{tabular}




\begin{tabular}{|cc|cccccc|} 
& Kappa & 0.84 & 0.82 & 0.84 & 0.89 & 0.85 & 0.9 \\
2005 & OA & $84.50 \%$ & $87.50 \%$ & $89.30 \%$ & $87.80 \%$ & $81.60 \%$ & $86.30 \%$ \\
& Kappa & 0.85 & 0.86 & 0.87 & 0.86 & 0.82 & 0.87 \\
2010 & OA & $86.10 \%$ & $84.30 \%$ & $89.70 \%$ & $82.30 \%$ & $88.10 \%$ & $84.90 \%$ \\
& Kappa & 0.84 & 0.83 & 0.88 & 0.82 & 0.87 & 0.85 \\
& OA & $88.90 \%$ & $90.20 \%$ & $85.60 \%$ & $83.90 \%$ & $87.60 \%$ & $89.30 \%$ \\
& Kappa & 0.87 & 0.91 & 0.86 & 0.82 & 0.86 & 0.9 \\
\hline
\end{tabular}

Table 3. Classification accuracy evaluation

\section{2 urban development pattern and pattern analysis}

\subsection{1 urban expansion and strength analysis}

Figure 4 shows the dynamic changes of the impervious layer in the major nodes along the South China Economic Belt. Vientiane, Hanoi and Phnom Penh are on the edge of the river. The expansion of these cities are by the river to inland. Due to the relatively developed economy, Bangkok, Kuala Lumpur and Singapore have the higher level of urbanization. The urban expansion mode is internal cluster development.
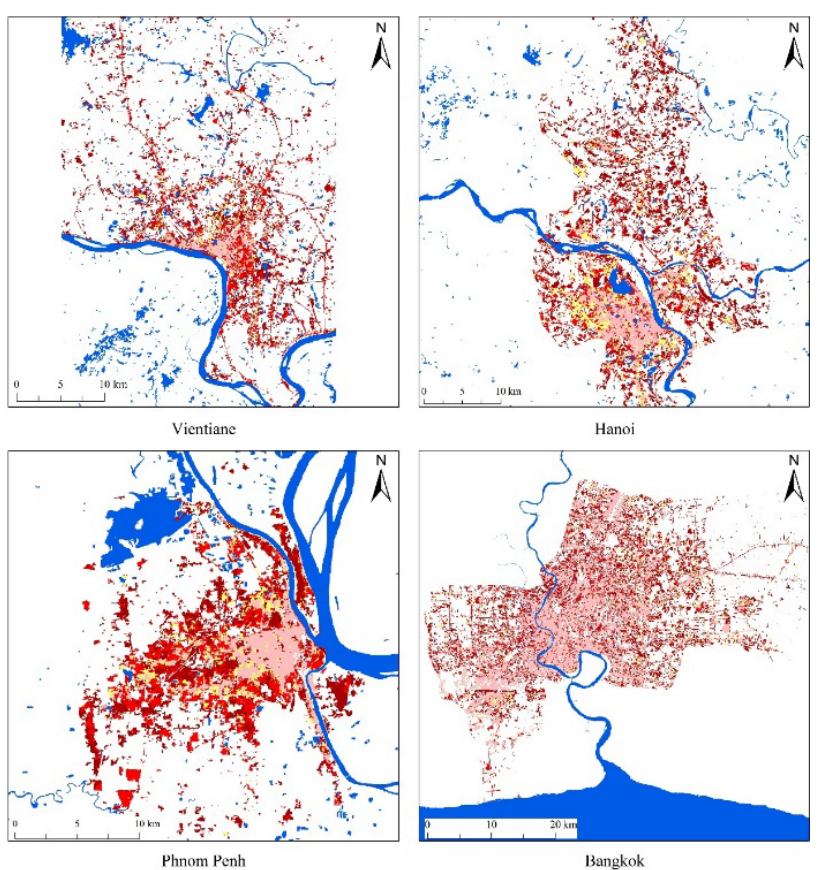

Phnom Penh
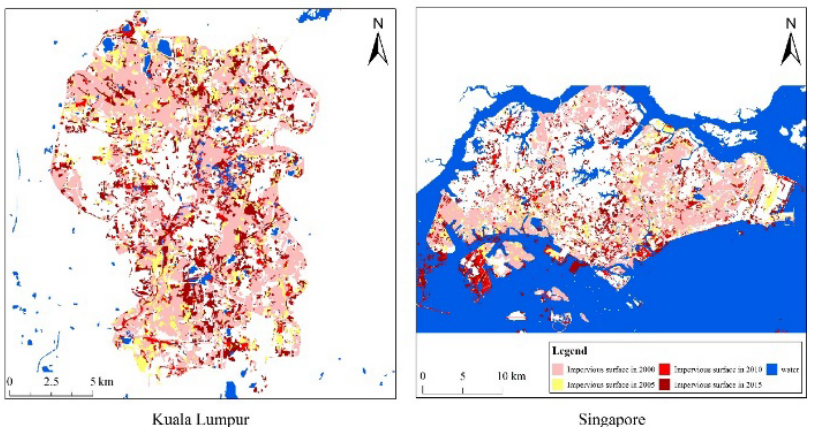

Figure. 4 the extension of impervious surface

Figure 5 shows a large percentage of each year in the area of city expansion, Figure 6 shows the average expansion rate of each city. It can be seen that these cities have different degrees of expansion during 2000-2015 from Figure 5. Among these cities, Vientiane has the largest urban expansion. Compared 
with 2000, the area is expanded nearly 8 times. Kuala Lumpur has the slowest rate of expansion. The area in 2015 was only 1.7 times the size of the area in 2000. As can be seen from Figure 6, Vientiane, Hanoi and Phnom Penh has maintained a high rate of urban expansion in 2000-2015, among those the average annual expansion rate of Vientiane reached 32\% during the year between 2005 and 2010. Bangkok, Kuala Lumpur and Singapore have a lower rate of urban expansion, with an average annual growth rate of less than $10 \%$ over the past 15 years. On the whole, these important node cities are in the rising stage of urban development.

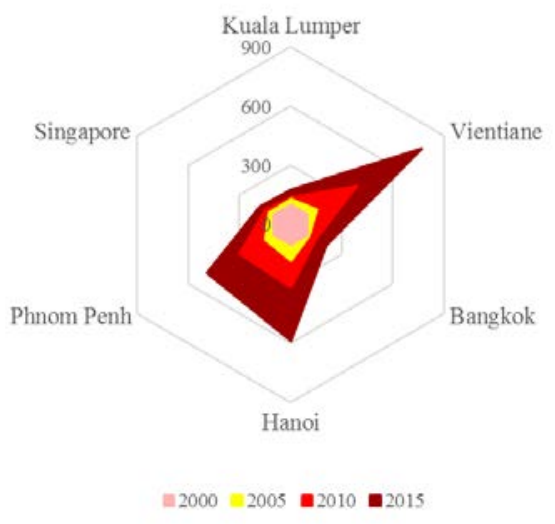

Figure 5. Percentage of area expansion

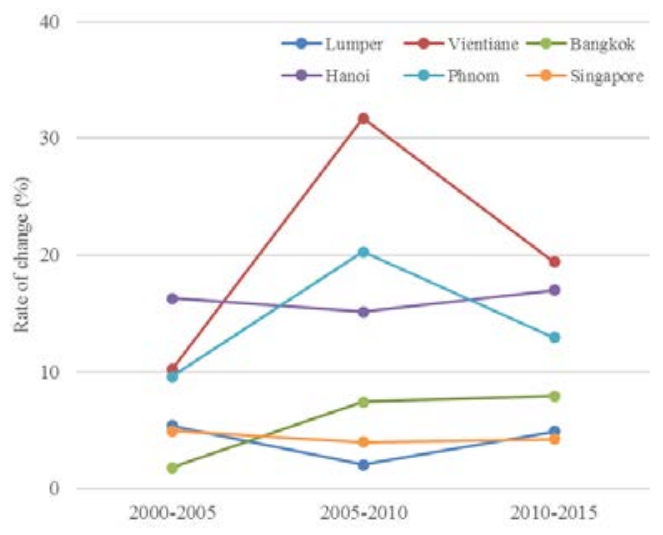

Figure 6. Expansion velocity

\subsection{2 urban landscape pattern analysis}

The changes of urban impervious surface and the change of landscape pattern indexes in 2000-2015 have been demonstrated. The urban expansion pattern was analysed based on the landscape metrics (Figure 7) and the change of urban impervious layer (Figure 4), the results are as follows.

LPI indicates the degree of influence of impermeable surface on the whole patch area, and the change of its value is helpful to determine the superiority of the impervious surface. In the period of 2000-2015.The LPI of each important node city showed an increasing trend year by year, indicating that the impervious area of the city is growing rapidly, and the urbanization process is obvious. The dominant position of impervious layer in landscape area is rising.

The ENN index indicates the nearest continental distance between impervious patches, and its size can reflect the aggregation degree of the impervious surface. Generally, the larger the ENN value, the distance between the classes of the same type is far away, and the distribution is discrete. On the contrary, it shows that the same type of block is close to each other, and it is a reunion distribution. During the period of 2000-2015, the fluctuation of ENN in Vientiane and Kuala Lumpur is relatively small, and the trend is decreasing, which indicates the development of impervious surface to spatial agglomeration. The increase of ENN in Hanoi shows that the impervious surface of Hanoi is increasing rapidly in space. The ENN of Phnom Penh, Bangkok and Singapore first increased and then decreased, indicating that the impervious surfaces of the 3 cities were dispersed and then developed.

SPLIT and DIVISION are both the index reflecting the aggregation and dispersion of patches in the landscape. During the period of 2000-2015, Vientiane and Phnom Penh were first increased and then decreased, which indicated that the urban expansion pattern was the spreading first and then gathering. Hanoi was first reduced and then increased, which indicated that the urban expansion mode was the first gathering and then spreading. While Bangkok, Kuala Lumpur and Singapore were gradually reduced, indicating that the pattern of urban expansion for the agglomeration development.

In a word, these 6 important cities along the Indochina Peninsula Economic Corridor have shown a rapid urbanization rate in the past 15 years. Due to the differences of the urban terrain, the development stage and other factors, the development model of the city is not the same. Therefore, it is necessary to analyse the type of urbanization expansion according to the stage of urban development.

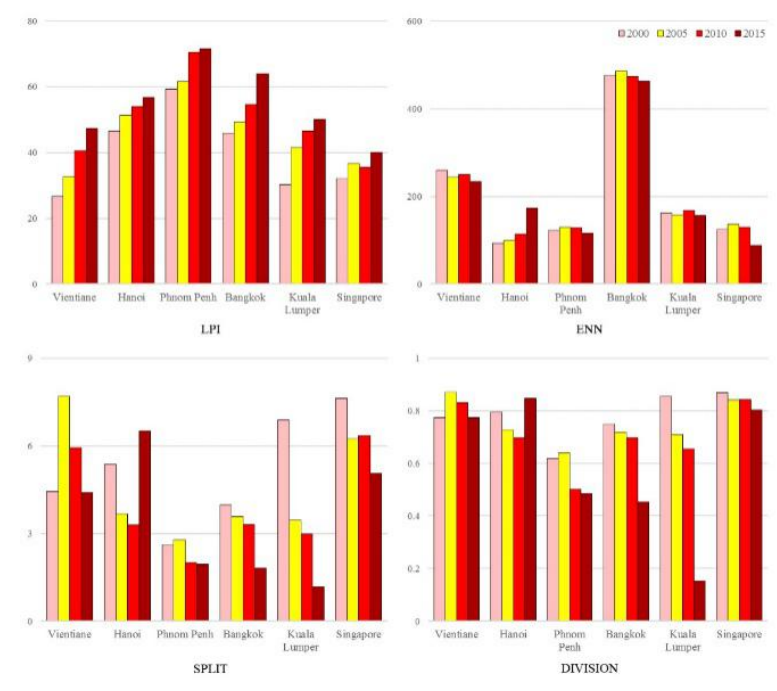

Figure 7. Landscape pattern metrics 


\section{CONCLUSIONS AND RECOMMENDATIONS}

The change of urban impervious surface reflects the economic development and urban development planning. In Southeast Asia, the ecological environment is good, the ecological resources are rich, the economic development potential is huge, and there is the global biodiversity conservation key region. Therefore, special attention should be paid to the coordinated development of regional economy and environment. In this study, the Landsat data of 6 important cities in the Indochina Peninsula economic belt were extracted by using the object-oriented random forest classification method to extract the urban impervious surface in the past 15 years. In this paper, we discuss the characteristics and laws of the spatial and temporal variation of urban expansion by the combination of visual analysis and quantitative calculation. The results show that in the past 15 years, the average annual growth rate of urban development and urban expansion are the highest, and the lowest in Kuala Lumpur. Vientiane and Phnom Penh urban expansion model are the first spread and then gathering; Hanoi urban expansion model is the first gathering and then spread, Bangkok, Kuala Lumpur and Singapore are the development of aggregation. It is fully explained that due to differences in urban development policies and urban development stages, different cities in the expansion mode is not the same. In general, most of the developed cities are clustered development, and the development of the city is the development of agglomeration after the first diffusion. The study has significant reference value for the real-time control of the rhythm of urban development in the Central South China Economic Zone, the rational planning of urban development, and the promotion of the coordinated development of economic environment.

\section{REFERENCES}

Breiman L., 1996. Bagging predictors[J]. Machine Learning, 1996, 24(2), pp. 123-140.

Breiman L., 2001. Random Forests[J]. Machine Learning, 45(1), pp. 5-32.

Che Q, Duan X et al., 2011. Urban Spatial Expansion Process, Pattern and Mechanism in Yangtze River Delta [J]. Acta Geographica Sinica, 66(4), pp. 446-456.

Chen L, Fu B, Wang J., 2001. Study on Land Use Change in a Small Typical Catchment in Loess Hilly Area: A Case Study in Danangou Catchment, Yan' an, Shanxi Province [J]. Scientia Geographica Sinica, 21(1), pp. 46-51.

Deng Y, Fan F, Chen R., 2012. Extraction and analysis of impervious surfaces based on a spectral un-mixing method using Pearl River Delta of China Landsat TM/ETM+ imagery from 1998 to 2008. [J]. Sensors, 12(2), pp. 1846.
Guo Y, Chi T, Peng L et al., 2016. Classification of GF-1 remote sensing Image based on random forests for urban land-use [J]. Bulletin of Surveying and Mapping, (5), pp. 73-76.

Ingram G K., 1997. Patterns of Metropolitan Development: What Have We Learned? [J]. Urban Studies, 35(7), pp. 1019-1035.

Liu D, Xia F., 2010. Assessing object-based classification: advantages and limitations[J]. Remote Sensing Letters, 1(4), pp. 187-194.

Liu X., 2015. The application of domestic satellite remote sensing of ecological and environmental protection in the construction of The Belt and Road " [J]. Satellite Application, (8), pp. 52-54.

Pan D, Domon G, Blois S D, et al., 1999. Temporal (19581993) and spatial patterns of land use changes in Haut-Saint-Laurent (Quebec, Canada) and their relation to landscape physical attributes[J]. Landscape Ecology, 14(1), pp. 35-52.

RIDD M.K.., 1995. Exploring a v-i-s (vegetation-impervious surface-soil) model for urban ecosystem analysis through remote sensing: comparative anatomy for cities. International Journal of Remote Sensing, 16(12), pp. 2165-2185.

Shi L, Zhang Z, Liu F et al . , 2015. Spatial expansion remote sensing monitoring of special economic zones from 1973 to 2013. Journal of Remote Sensing, 19 (6), pp.1030-1039.

Taubenböck. H, Esch. T, Felbier. A, et al., 2012. Monitoring urbanization in mega cities from space[J]. Remote Sensing of Environment, 117(2012-02), pp. 162-176.

Wang L, Duan X., 2010. The Expansion of Urbanization Area in Yangtze River Delta [J]. Scientia Geographica Sinica, 16(5), pp. 702-709.

Wang L, Feng C.,2016. Spatial expansion pattern and its driving dynamics of Beijing-Tianjin-Hebei metropolitan region: Based on night-time light data [J]. Acta Geographica Sinica, (12), pp. 2155-2169.

Wolpert D H, Macready W G.,1999. An Efficient Method To Estimate Bagging's Generalization Error[C]// Santa Fe Institute, pp. 41-55.

Zang S, Wu C, Liu H, et al., 2010. Impact of urbanization on natural ecosystem service values: a comparative study[J]. Environmental Monitoring \& Assessment, 179(1-4), pp. 575-588. 